\title{
ESCALA DE PROJETOS DE VIDA PARA ADOLESCENTES: EVIDÊNCIAS DE VALIDADE DE CONTEÚDO
}

\author{
Jessica Particelli Gobbo \\ Pontifícia Universidade Católica de Campinas - PUC/ CAMPINAS \\ Tatiana de Cássia Nakano \\ Pontifícia Universidade Católica de Campinas - PUC/ CAMPINAS \\ Letícia Lovato Dellazzana-Zanon \\ Pontifícia Universidade Católica de Campinas - PUC/ CAMPINAS
}

\begin{abstract}
Resumo
A temática dos projetos de vida de adolescentes tem se mostrado um tema relevante na Psicologia, especificamente, na Psicologia do Desenvolvimento. Diante da lacuna de instrumentais que tenham esse objetivo, o presente estudo consistiu na investigação das evidências de validade de conteúdo da Escala de Projetos de Vida para Adolescentes (EPVA), em desenvolvimento. Para isso, cinco peritos no assunto atuaram como juízes, classificando os 116 itens do instrumento em sete dimensões: relacionamentos afetivos, estudo, trabalho, aspirações positivas, bens materiais, religião/espiritualidade e sentido da vida. A análise dos coeficientes de concordância demonstraram a adequação dos itens, sendo possível manter $88 \%$ da escala inicial. As análises dos coeficientes de Kappa evidenciaram índice de concordância acima de 0,75 para todos os juízes. Os resultados apontaram para a adequação da escala ao construto e dimensões que pretende avaliar.

Palavras-chave: projetos de vida; validade de conteúdo; avaliação psicológica; coeficiente kappa.
\end{abstract}

\section{LIFE'S PROJECTS SCALE FOR ADOLESCENTS: EVIDENCE OF CONTENT VALIDITY}

\begin{abstract}
The thematic of the life projects of adolescents has been shown relevant in Psychology, specifically in Developmental Psychology. Faced with the lack of instruments that have this objective, the present study consisted in the investigation of the evidence of content validity of the Scale of Life Projects for Adolescents (EPVA), in development. Five experts on the subject acted as judges, classifying the 116 items of the instrument in seven dimensions: affective relationships, study, work, positive aspirations, material goods, religion / spirituality and meaning of life. The analysis of the coefficients of agreement showed the adequacy of the items, being possible to maintain $88 \%$ of the initial scale. Analysis of Kappa coefficient showed a concordance index above 0.75 for all judges. The results pointed to the adequacy of the scale to the construct and dimensions that it intends to evaluate.
\end{abstract}

Keywords: life's projects; content validity; psychological assessment; kappa coefficient. 


\title{
ESCALA DE PROYECTOS DE VIDA PARA LOS ADOLESCENTES: VALIDEZ DE CONTENIDO
}

\begin{abstract}
Resumen
La temática de los proyectos de vida de adolescentes se ha mostrado un tema relevante en la Psicología, específicamente, en la Psicología del Desarrollo. Ante la falta de instrumentos que tienen ese objetivo, el presente estudio consistió en la investigación de las evidencias de validez de contenido de la Escala de Proyectos de Vida para Adolescentes (EPVA), en desarrollo. Para ello, cinco expertos en el asunto actuaron como jueces, clasificando los 116 ítems del instrumento en siete dimensiones: relaciones afectivas, estudio, trabajo, aspiraciones positivas, bienes materiales, religión / espiritualidad y sentido de la vida. El análisis de los coeficientes de concordancia demostró la adecuación de los ítems, siendo posible mantener el 88\% de la escala inicial. Los análisis de los coeficientes de Kappa evidenciaron un índice de concordancia superior a 0,75 para todos los jueces. Los resultados apuntaron a la adecuación de la escala al constructo y dimensiones que ésta pretende evaluar.

Palabras clave: proyectos de vida; validez de contenido; evaluación psicológica; coeficiente kappa.
\end{abstract}

\section{INTRODUÇÃO}

A adolescência tem sido considerada uma fase do desenvolvimento humano propícia para a construção dos projetos de vida, tanto por autores clássicos (Erikson, 1968/1976; Piaget, 1964/2007), como por contemporâneos (Bronk, 2014; Damon, 2009; D'Aurea-Tardeli, 2010; Dellazzana-Zanon \& Freitas, 2015; Furlani \& Bomfim, 2010; Leão, Dayrell, \& Reis, 2011). Nessa fase do desenvolvimento, o sujeito começa a se inserir no mundo dos adultos e passa a julgá-los em um plano de igualdade e de total reciprocidade (Piaget, 1964/2007), mostrando-se, ainda um sujeito em formação, capaz de pensar no seu futuro dentro de uma determinada sociedade. Do mesmo modo o adolescente é capaz de pensar em suas atividades momentâneas dentro de um programa de vida para desenvolver atividades futuras (Inhelder \& Piaget, 1958/1976).

Ainda que o estudo dos projetos de vida venha se mostrando, cada vez mais, valorizado na atualidade, a ausência de consenso sobre sua definição, entre os autores interessados na temática se faz presente (Dellazzana-Zanon \& Freitas, 2015). Algumas definições clássicas entendem projeto de vida como resultado do desenvolvimento das estruturas formais (pensamento formal) que possibilitam o adolescente a raciocinar sobre o seu futuro, ou seja, de formular hipóteses que ultrapassam a fronteira do real (Inhelder \& Piaget, 1958/1976) ou ainda como a base para a resolução bem sucedida da crise de identidade do adolescente (Erikson, 1968/1976). Por outro lado, definições mais contemporâneas entendem este construto como "uma intenção estável e generalizada de alcançar algo que seja significativo para o eu e que gere um compromisso produtivo para algum aspecto além do eu" (Damon, Menon, \& Bronk, 2003, p. 121). Assim, embora existam diferentes definições sobre o que seria um projeto de vida, nesta pesquisa ele é compreendido como "o conjunto de metas que um indivíduo estabelece para si próprio e o desenvolvimento 
dessas metas ao longo da vida, o qual: (a) é construído com base no contexto cultural em que este indivíduo está inserido, (b) dá sentido para a sua vida e (c) pode ou não incluir outras pessoas" (Dellazzana-Zanon, 2016, p. 9).

Pesquisas têm demonstrado que a elaboração de um projeto de vida e, consequentemente, da projeção de um futuro, parece trazer benefícios para a pessoa, como a realização e satisfação pessoal, garantia de mais resiliência em momentos difíceis, bem como proteção perante possíveis comportamentos autodestrutivos (Bronk, 2014; Damon, 2009). Da mesma forma, adolescentes que não constroem projetos de vida tendem a apresentar mais comportamentos de risco associados à violência, doenças sexualmente transmissíveis, gravidez precoce e uso de drogas (Bronk, 2014; Damon, 1995, Farias Júnior et al., 2009; Feijó \& Oliveira, 2001). Tais constatações justificam a necessidade de aprofundamento e investigação dessa temática nos dias atuais, visto que, de forma bastante comum, a adolescência tem sido associada a um período em que o adolescente passa muito tempo à deriva, ao invés de pensar sobre o seu futuro (Damon, 2012; La Taille, 2009).

Uma das dificuldades na temática, além do número ainda pequeno de pesquisadores dedicados a ela e à ausência de consenso em sua definição, citados anteriormente, está relacionada à sua avaliação. Internacionalmente, o cenário aponta para a existência de uma grande variedade de métodos quantitativos, identificada após trabalho de revisão não sistemática dos instrumentos utilizados, feita nos Estados Unidos. Dentre eles, três instrumentos internacionais específicos para avaliar adolescentes se destacam: (a) Existence of Purpose in Life subscale (EPIL; Law, 2012) for early adolescence, (b) Life Purpose Questionnaire - Adolescent version (LPQ-A; Hablas \& Hutzell, 1982) e (c) Revised Youth Purpose Survey (Bundick et al., 2006). A escala EPIL é derivada do Purpose in Life Test (PIL, Crumbaugh, 1968; Crumbaugh \& Maholick, 1964) e é o instrumento que mais passou por estudos de suas validades psicométricas. A escala $L P Q-A$ é também um instrumento derivado da PIL e sua versão para adolescentes é composta por dois itens da versão original para adultos. Ela tem sido usada em adolescentes envolvidos com álcool e drogas, porém possui poucos estudos para investigar sua validade psicométrica. Por fim, a Revised Youth Purpose Survey (Bundick et al., 2006) é uma escala multidimensional desenvolvida para avaliar a procura por projetos de vida, a presença de projetos, o engajamento no trabalho e a centralidade e o significado do projeto de vida. Destaca-se o fato de que relatos de uso de tais instrumentos em pesquisas brasileiras são encontrados, ainda que somente a tradução dos mesmos tenha sido feita, fazendo-se notar a ausência de estudos de investigação de suas evidências de validade para uso na nossa população. Por outro lado, enquanto no contexto internacional se fazem disponíveis diferentes formas de avaliar projetos de vida, o mesmo não acontece no Brasil, ou seja, faltam instrumentos quantitativos para acessar projetos de vida de adolescentes. 
A preferência por instrumentos qualitativos foi notada em pesquisas nacionais (do tipo entrevistas, questões de autorrelato e grupos de discussão), não se tendo notícia da existência de instrumentos quantitativos específicos para avaliar projetos de vida de adolescentes no Brasil. Dessa forma, considerando-se que: (a) a adolescência é um período fértil do desenvolvimento humano para a construção dos projetos de vida; (b) ter um projeto de vida pode trazer benefícios à vida do adolescente e (c) não há um instrumento padronizado para investigar projetos de vida quantitativamente no Brasil, foi iniciado o processo de construção da Escala de Projetos de Vida para Adolescentes (EPVA). A escala foi pensada para voltar-se à compreensão de como se caracterizam os projetos de vida dos adolescentes, a identificação de se estão pensando ou não nesses projetos e, em caso positivo, em quais dimensões os estruturam. A relevância da avaliação dos projetos de vida se ampara na constatação de que adolescentes que têm projetos de vida adquirem mais atitudes positivas perante a vida, assim como desenvolvem mais resultados desejáveis perante a sociedade e sobre si mesmo como: comportamento pró-social, compromisso moral, realização e autoestima elevada (Damon et al., 2003).

Para tanto, a escala foi construída de forma a abarcar os diferentes aspectos envolvidos no construto, após consulta à literatura científica: estudo, trabalho, família, aquisição de bens materiais, religiosidade, relações interpessoais e qualidade de vida (Araújo, Arantes, Danza, Pinheiro \& Garbin, 2016; Bock \& Liebesny, 2003; Cardoso \& Cocco, 2003; D’ Aurea-Tardeli, 2008; Furlani \& Bomfim, 2010; Graf \& Diogo, 2009; Gonçalves et al., 2008; Miranda, 2007; Parede \& Pecora, 2004; Pratta \& Santos, 2007; Santos, 2002). Seus itens iniciais foram redigidos de forma a contemplarem, teoricamente, tais aspectos.

Assim, a presente pesquisa tem como objetivo apresentar um primeiro estudo de evidências de validade de conteúdo da Escala de Projetos de Vida para Adolescentes (EPVA), com o propósito de identificar e reconhecer aspectos fortes e fracos do instrumento antes da sua aplicação empírica. Tal tipo de validade se refere à análise do conjunto de itens que elegeu para compor o teste e se eles são, efetivamente, representativos dos comportamentos relacionados ao construto latente em questão (Pacico \& Hutz, 2015). A avaliação sobre a pertinência dos itens ao construto que representam pode ser feita por juízes (Pasquali, 2010), tendo sido, tal método, utilizado no presente estudo.

\section{MÉTODO}

\section{Participantes}

A amostra foi composta por cinco juízes independentes, três do sexo feminino e dois do sexo masculino. Todos os juízes eram psicólogos e três 
também eram professores universitários. O nível educacional dos juízes participantes foi dois juízes pós-doutores, dois doutores e um especialista.

\section{Instrumento}

Escala de Projetos de Vida para Adolescentes (EPVA). A escala constitui-se em um instrumento para avaliação dos projetos de vida dos adolescentes organizada em sete dimensões. As dimensões que compõem a EPVA são apresentadas na Tabela 1.

Tabela 1. Definições das dimensões da EPVA

\begin{tabular}{|c|c|c|}
\hline Dimensão & $\begin{array}{l}\mathrm{N}^{\circ} \text { de } \\
\text { itens }\end{array}$ & Definição \\
\hline 1 & 20 & $\begin{array}{l}\text { Projetos relacionados a iniciar, manter, ou intensificar } \\
\text { relacionamentos afetivos. Essa dimensão é composta pelas } \\
\text { subcategorias: (a) constituir família, com ou sem filhos; (b) } \\
\text { convivência com a família de origem, (c) namorar e (d) } \\
\text { projetos que envolvam ajudar algum familiar. }\end{array}$ \\
\hline 2 & 19 & $\begin{array}{l}\text { Projetos relacionados à continuidade dos estudos. Essa } \\
\text { dimensão é composta pelas subcategorias: (a) terminar os } \\
\text { estudos e (b) fazer faculdade. }\end{array}$ \\
\hline 3 & 17 & $\begin{array}{l}\text { Projetos relacionados a exercer uma profissão ou ter um } \\
\text { emprego. }\end{array}$ \\
\hline 4 & 16 & $\begin{array}{l}\text { Projetos referentes à vontade de se melhorar enquanto } \\
\text { pessoa ao longo do tempo. Incluem-se projetos que } \\
\text { indiquem a vontade de se tornar uma pessoa melhor que } \\
\text { faça, de alguma forma, diferença na vida de outras pessoas } \\
\text { e na sociedade. }\end{array}$ \\
\hline 5 & 17 & $\begin{array}{l}\text { Projetos relacionados à aquisição de bens materiais e ao } \\
\text { desejo de melhoria da condição financeira. }\end{array}$ \\
\hline 6 & 14 & $\begin{array}{l}\text { Projetos relacionados à satisfação com a conexão pessoal } \\
\text { com Deus ou com algo que se considere como absoluto. }\end{array}$ \\
\hline 7 & 13 & $\begin{array}{l}\text { Projetos relacionados ao sentido da vida independentemente } \\
\text { de uma referência religiosa. Incluem nessa categoria } \\
\text { também, projetos relacionados à ausência do sentido da } \\
\text { vida. }\end{array}$ \\
\hline
\end{tabular}

Nota. Na coluna Dimensão: 1. Relacionamentos Afetivos; 2. Estudo; 3. Trabalho; 4. Aspirações Positivas; 5. Bens Materiais; 6. Religião/Espiritualidade; 7. Sentido da Vida.

Nessa fase da pesquisa, os juízes receberam um documento, contendo os 116 itens que compunham a versão da EPVA a ser avaliada (conforme descrição na Tabela 1), e uma tabela contendo as definições das sete dimensões. 


\section{Procedimentos}

Após a aprovação do projeto pelo Comitê de Ética em Pesquisa com Seres Humanos da Pontifícia Universidade Católica de Campinas (CAAE 52898416.9.0000.5481), cada juiz assinou o Termo de Consentimento Livre e Esclarecido. Posteriormente, um formulário de avaliação foi elaborado e enviado aos juízes, por e-mail, no qual inicialmente o objetivo da pesquisa era fornecido, explicando-se a tarefa a ser desenvolvida. Dois diferentes julgamentos deveriam ser feitos pelo juiz. Primeiramente ele deveria analisar cada item considerandose três aspectos: (a) clareza, (b) pertinência prática e (c) pertinência teórica. Em segundo lugar, era solicitada a classificação de cada item em uma das sete dimensões criadas. Com a finalidade de evitar que itens de uma mesma área estivessem agrupados, eles foram organizados em uma única lista e apresentados de forma aleatória, de modo que não fosse possível identificar os domínios a que pertenciam por similaridade ou proximidade. Uma quinta coluna, nomeada de observações, também foi incluída no formulário, na qual era possível que o juiz colocasse comentários que achasse pertinente sobre o item em questão.

Após o recebimento das tarefas concluídas, as pesquisadoras fizeram o levantamento de respostas dos juízes para cada um dos 116 itens, utilizando-se para análise dos dados de dois diferentes procedimentos: porcentagem de concordância e o cálculo do coeficiente Kappa. A opção pela utilização conjunta dos dois procedimentos baseou-se na constatação de Perroca e Gaidzinski (2003), segundo os quais, apesar de largamente utilizado para o estudo de confiabilidade, o coeficiente Kappa apresenta limitações na medida em que não fornece informações a respeito da estrutura de concordância e discordância, muitas vezes não considerando aspectos importantes presentes nos dados. Dessa forma, não deve ser utilizado indiscriminadamente como uma única medida de concordância, devendo-se incorporar outras abordagens com o objetivo de complementar a análise. Buscando sanar essa lacuna, um segundo método foi empregado no presente estudo, mais simples, de verificação da concordância interobservadores, por meio do cálculo da porcentagem de concordância entre juízes independentes, de maneira que os dois métodos empregados se complementaram.

A análise da porcentagem de concordância seguiu, como parâmetros de interpretação, os valores sugeridos por Landis e Koch (1977): concordância quase perfeita (entre 0,80 e 1,00), concordância substancial $(0,60$ a 0,80), concordância moderada $(0,40$ a 0,60$)$, concordância regular $(0,20$ a 0,40$)$ e concordância discreta $(0,00$ a 0,20$)$. Já para o coeficiente Kappa, os valores seguiram as recomendações de Fleiss, Levin e Park (2003): (a) acima de 0,75, concordância excelente, (b) entre 0,40 e 0,75, concordância satisfatória e (c) abaixo de 0,40 , concordância insatisfatória. 
Como critérios para que os itens fossem considerados adequados e, portanto, mantidos na escala, determinou-se que os juízes deveriam ter concordância acima de $80 \%$, conforme a recomendação da literatura científica (Pasquali, 2010) em, pelo menos dois dos três primeiros critérios avaliados (clareza, pertinência prática e pertinência teórica), sem considerar, em um primeiro momento, a dimensão. Aqueles itens que atenderam a esse critério foram mantidos na escala e os que ficaram abaixo foram automaticamente excluídos. Posteriormente, os itens que passaram pelo primeiro filtro, foram analisados em termos de percentual de concordância dos juízes, considerando-se então a dimensão.

\section{RESULTADOS}

Para fins de análise, os itens foram divididos de acordo com a dimensão avaliada. Assim sendo, a Tabela 2 apresenta os resultados da proporção total de concordância entre os juízes para cada item correspondente a cada uma das dimensões. Salienta-se que a Tabela apresenta os dados da análise acerca dos critérios qualitativos (clareza, pertinência prática e pertinência teórica), bem como a dimensão apontada pelos juízes e a dimensão conferida ao item.

Aqueles índices que não apresentaram porcentagem de concordância ideal (acima de 80\%), estão indicados por meio de fundo cinza, sendo importante destacar que, caso tal situação ocorresse em relação ao julgamento da dimensão a que pertence o item, o mesmo não foi atribuído a nenhuma delas, sendo excluído ou reformulado.

Tabela 2. Proporção total de concordância entre os juízes para os itens em cada uma das dimensões

\begin{tabular}{ccccccc}
\hline Dimensão ideal & Item & Clareza & $\begin{array}{c}\text { Pertinência } \\
\text { prática }\end{array}$ & $\begin{array}{c}\text { Pertinência } \\
\text { teórica }\end{array}$ & Dimensão & $\begin{array}{c}\text { Dimensão } \\
\text { conferida }\end{array}$ \\
\hline 1 & 17 & 60 & 80 & 80 & 80 & 1 \\
& 31 & 80 & 80 & 80 & 80 & 1 \\
& 61 & 100 & 100 & 100 & 100 & 1 \\
41 & 80 & 80 & 80 & 60 & 1 \\
48 & 100 & 100 & 100 & 80 & 1 \\
& 23 & 80 & 80 & 80 & 80 & 6 \\
98 & 100 & 100 & 100 & 100 & 1 \\
& 86 & 100 & 100 & 100 & 100 & 1 \\
52 & 80 & 80 & 80 & 100 & 1 \\
& 108 & 100 & 100 & 100 & 60 & 1 \\
& 2 & 80 & 80 & 100 & 80 & 1
\end{tabular}




\begin{tabular}{|c|c|c|c|c|c|c|}
\hline & 102 & 100 & 100 & 100 & 100 & 1 \\
\hline & 25 & 100 & 80 & 80 & 60 & 1 \\
\hline & 76 & 80 & 100 & 100 & 100 & 1 \\
\hline & 4 & 80 & 100 & 80 & 100 & 1 \\
\hline & 53 & 100 & 100 & 100 & 100 & 1 \\
\hline & 70 & 80 & 100 & 100 & 100 & 1 \\
\hline & 18 & 80 & 100 & 100 & 100 & 1 \\
\hline & 10 & 100 & 100 & 80 & 80 & 1 \\
\hline & 75 & 100 & 100 & 100 & 100 & 1 \\
\hline \multirow{19}{*}{2} & 3 & 80 & 60 & 40 & 100 & \\
\hline & 24 & 100 & 100 & 100 & 100 & 2 \\
\hline & 47 & 80 & 80 & 80 & 80 & 2 \\
\hline & 96 & 100 & 80 & 80 & 100 & 2 \\
\hline & 117 & 100 & 100 & 100 & 100 & 2 \\
\hline & 107 & 100 & 100 & 100 & 100 & 2 \\
\hline & 62 & 100 & 100 & 100 & 100 & 2 \\
\hline & 87 & 80 & 80 & 80 & 100 & 2 \\
\hline & 115 & 80 & 100 & 100 & 80 & 2 \\
\hline & 36 & 80 & 80 & 80 & 60 & \\
\hline & 68 & 80 & 100 & 100 & 80 & 2 \\
\hline & 83 & 60 & 60 & 60 & 40 & \\
\hline & 9 & 60 & 80 & 80 & 100 & 2 \\
\hline & 63 & 80 & 100 & 100 & 80 & 2 \\
\hline & 113 & 60 & 100 & 100 & 60 & \\
\hline & 99 & 80 & 100 & 100 & 60 & \\
\hline & 74 & 100 & 100 & 100 & 100 & 2 \\
\hline & 92 & 80 & 80 & 80 & 100 & 2 \\
\hline & 69 & 80 & 80 & 80 & 80 & 2 \\
\hline \multirow{13}{*}{3} & 11 & 80 & 80 & 80 & 100 & 3 \\
\hline & 35 & 100 & 100 & 100 & 80 & 3 \\
\hline & 116 & 80 & 100 & 100 & 40 & \\
\hline & 80 & 100 & 100 & 100 & 80 & 3 \\
\hline & 85 & 60 & 60 & 60 & 60 & \\
\hline & 64 & 100 & 100 & 100 & 100 & 3 \\
\hline & 82 & 80 & 100 & 100 & 100 & 3 \\
\hline & 103 & 100 & 100 & 100 & 60 & \\
\hline & 8 & 80 & 100 & 100 & 60 & \\
\hline & 97 & 80 & 80 & 80 & 80 & 3 \\
\hline & 114 & 100 & 100 & 100 & 100 & 3 \\
\hline & 46 & 100 & 80 & 80 & 100 & 3 \\
\hline & 22 & 80 & 80 & 80 & 80 & 3 \\
\hline
\end{tabular}




\begin{tabular}{|c|c|c|c|c|c|c|}
\hline & 56 & 100 & 100 & 100 & 100 & 3 \\
\hline & 42 & 100 & 100 & 100 & 100 & 3 \\
\hline & 21 & 80 & 60 & 60 & 80 & \\
\hline & 39 & 80 & 80 & 80 & 80 & 3 \\
\hline & 26 & 100 & 100 & 100 & 80 & 4 \\
\hline & 55 & 100 & 100 & 100 & 60 & \\
\hline & 43 & 100 & 100 & 100 & 60 & \\
\hline & 49 & 100 & 80 & 100 & 100 & 4 \\
\hline & 54 & 80 & 80 & 80 & 80 & 4 \\
\hline & 112 & 80 & 100 & 100 & 80 & 4 \\
\hline 4 & 38 & 100 & 100 & 100 & 80 & 4 \\
\hline 4 & 93 & 100 & 100 & 100 & 80 & 4 \\
\hline & 88 & 80 & 80 & 100 & 80 & 4 \\
\hline & 44 & 40 & 100 & 100 & 60 & \\
\hline & 73 & 80 & 100 & 100 & 80 & 4 \\
\hline & 104 & 60 & 80 & 80 & 80 & 4 \\
\hline & 20 & 100 & 100 & 100 & 80 & 4 \\
\hline & 27 & 60 & 60 & 60 & 60 & \\
\hline & 66 & 100 & 100 & 100 & 80 & 4 \\
\hline & 45 & 80 & 80 & 80 & 100 & 5 \\
\hline & 84 & 60 & 80 & 80 & 80 & 5 \\
\hline & 14 & 60 & 80 & 80 & 80 & 5 \\
\hline & 94 & 80 & 100 & 100 & 100 & 5 \\
\hline & 100 & 100 & 100 & 100 & 100 & 5 \\
\hline & 1 & 100 & 60 & 80 & 80 & 5 \\
\hline & 28 & 100 & 100 & 100 & 100 & 5 \\
\hline & 72 & 100 & 80 & 100 & 100 & 5 \\
\hline 5 & 50 & 80 & 80 & 80 & 80 & 5 \\
\hline & 77 & 80 & 100 & 100 & 80 & 5 \\
\hline & 57 & 80 & 80 & 40 & 100 & 5 \\
\hline & 111 & 100 & 100 & 100 & 100 & 5 \\
\hline & 67 & 100 & 100 & 100 & 100 & 5 \\
\hline & 101 & 80 & 60 & 60 & 80 & 5 \\
\hline & 89 & 100 & 100 & 100 & 100 & 5 \\
\hline & 105 & 80 & 80 & 80 & 60 & \\
\hline & 19 & 60 & 40 & 60 & 100 & \\
\hline & 15 & 60 & 60 & 60 & 80 & \\
\hline & 29 & 60 & 100 & 100 & 100 & 6 \\
\hline 6 & 51 & 60 & 60 & 60 & 60 & \\
\hline & 58 & 100 & 100 & 100 & 100 & 6 \\
\hline & 34 & 80 & 80 & 80 & 100 & 6 \\
\hline
\end{tabular}




\begin{tabular}{ccccccc} 
& 81 & 60 & 100 & 100 & 100 & 6 \\
59 & 80 & 80 & 80 & 60 & \\
110 & 100 & 100 & 100 & 100 & 6 \\
37 & 80 & 80 & 80 & 80 & 6 \\
5 & 80 & 80 & 80 & 100 & 6 \\
90 & 60 & 60 & 80 & 100 & 6 \\
79 & 60 & 80 & 80 & 100 & 6 \\
60 & 80 & 80 & 100 & 100 & 6 \\
32 & 100 & 80 & 80 & 100 & 6 \\
\hline 30 & 80 & 100 & 100 & 100 & 7 \\
16 & 60 & 60 & 60 & 100 & \\
7 & 80 & 40 & 20 & 60 & \\
95 & 80 & 80 & 40 & 60 & \\
91 & 80 & 80 & 40 & 60 & \\
71 & 80 & 80 & 80 & 60 & \\
109 & 60 & 60 & 40 & 40 & \\
78 & 80 & 80 & 100 & 100 & 7 \\
106 & 60 & 60 & 40 & 40 & \\
33 & 100 & 100 & 80 & 40 & \\
40 & 80 & 80 & 60 & 40 & \\
65 & 80 & 60 & 40 & 60 & \\
6 & 100 & 80 & 80 & 40 & \\
\hline
\end{tabular}

Nota. Na coluna Dimensão ideal: 1. Relacionamentos Afetivos; 2. Estudo; 3. Trabalho; 4. Aspirações Positivas; 5. Bens Materiais; 6. Religião/Espiritualidade; 7. Sentido da Vida.

Os resultados da dimensão Relacionamentos Afetivos indicaram que um dos itens (item 17) não apresentou concordância no critério de clareza. Como houve concordância nos outros dois critérios, o item foi mantido, conforme critérios definidos anteriormente. Em relação à dimensão, dos 20 itens analisados, 11 apresentaram concordância de 100\% (55\%), seis apresentaram concordância de $80 \%(30 \%)$ e três apresentaram concordância de $60 \%(15 \%)$. Assim, tomando-se o critério estabelecido ( $80 \%$ de concordância), a maior parte deles foi mantida após essa análise ( $85 \%)$. Os três itens que não apresentaram valores satisfatórios foram reescritos e enviados novamente para os juízes. 0 conteúdo presente nesses itens referia-se a ajudar os familiares financeiramente quando eles estiverem mais velhos e a passar os valores para a família que iria construir. Houve apenas um caso de ausência de resposta de um juiz para o item 10 (caso que representaria que o juiz não conseguiu identificar a dimensão do item). 
Os resultados da dimensão Estudo indicaram que os itens 3 e 83 apresentaram concordância entre os juízes menor do que $80 \%$ em mais de um critério (pertinência prática e teórica para o item 3 e nos três critérios no caso do item 83), de modo que eles foram excluídos. Já os itens 9 e 113 apresentaram concordância adequada somente no critério de clareza, sendo mantidos. Em relação à dimensão, dos 19 itens analisados, 10 apresentaram concordância de $100 \%$ para a dimensão (52\%), cinco apresentaram concordância de $80 \%$ (26\%), três apresentaram concordância de $60 \%$ (15\%) e um apresentou concordância de $40 \%$ (5\%). Assim, tomando-se o critério de $80 \%$ de concordância, a maior parte deles foi mantida após essa análise (78\%). Os três itens que não apresentaram valores satisfatórios, foram reescritos e enviados novamente para os juízes. O conteúdo desses itens referia-se a melhorar a condição financeira por meio dos estudos. Houve três casos de ausência de resposta para os itens 36,69 e 83.

A terceira dimensão avaliada, Trabalho, apresentou um item com problema nos quatro quesitos avaliados (item 85) e um item com concordância menor do que a desejada em dois critérios (pertinência prática e teórica), tendo sido excluídos da escala. Em relação à dimensão, dos 17 itens analisados, sete apresentaram concordância de $100 \%$ (41\%), cinco apresentaram concordância de $80 \%$ (35\%), três apresentaram concordância de 60\% (17\%) e um apresentou concordância de $40 \%$ (5\%). Assim, tomando-se o critério de $80 \%$ de concordância, a maior parte deles foi mantida após essa análise (76\%). Os quatro itens que não apresentaram valores satisfatórios foram reescritos e enviados novamente para os juízes. Houve, também, três casos de ausência de resposta (caso que o juiz não conseguiu identificar a dimensão do item).

$\mathrm{Na}$ dimensão Aspirações Positivas, o item 27 apresentou ausência de concordância satisfatória em todos os critérios avaliados, sendo excluído. Os itens 44 e 104 somente no critério de clareza, sendo, portanto, mantidos. Tomando-se a análise da concordância dos juízes em relação à dimensão, os resultados indicaram que, dos 16 itens analisados, apenas um item apresentou concordância de 100\% (6\%), 11 apresentaram concordância de 80\% (69\%), e quatro apresentaram concordância de $60 \%$ (25\%). Assim, a maior parte deles foi mantida (75\%). Os itens que não apresentaram valores satisfatórios (itens 43 envolvendo o desejo de fazer alguma diferença no mundo, 44 - contemplava o desejo de que a vida estivesse melhor do que ela é atualmente e 55 - desejo de ser mais feliz do que é hoje) foram reescritos e enviados novamente para os juízes. Note-se que, nessa dimensão, o item 44 foi classificado pela maioria dos juízes na dimensão Sentido da Vida. Houve, também, um caso de ausência de resposta de um dos juízes para o item 104.

$\mathrm{Na}$ dimensão Bens Materiais, quatro itens apresentaram valores mais baixos do que o desejado em algum dos critérios (itens 84, 14, 1 e 57), um deles apresentou em dois critérios (item 101) e o item 19 nos três critérios. Assim, os 
primeiros itens foram mantidos e os dois últimos, excluídos. Analisando-se a dimensão, dos 17 itens, nove apresentaram concordância de 100\% (52\%) e seis apresentaram concordância de 80\% (35\%). Somente o item 105 apresentou concordância de $60 \%$. Tal item envolvia a ausência de desejo de ter mais coisas do que a pessoa já possui hoje. Note-se que, nessa dimensão, o item 57 , sobre o projeto de morar fora do país, não foi avaliado por nenhum dos juízes em relação à dimensão. Outros itens também não foram classificados em nenhuma dimensão por, pelo menos, um dos juízes: 50, 84, 101 e 105.

$\mathrm{Na}$ dimensão Religião/Espiritualidade, os itens 15, 51 e 90 apresentaram problemas em relação aos critérios estipulados para o primeiro filtro (clareza, pertinência prática e teórica). Por tal motivo, foram excluídos. Já os itens 29, 81 e 79 apresentaram concordância menor do que a desejada somente no quesito clareza, tendo sido mantidos na segunda análise, relacionada à dimensão a que pertence 0 item. Dos 12 itens analisados, nove apresentaram concordância de $100 \%$ (75\%), dois apresentaram concordância de $80 \%$ (14\%) e um apresentou concordância de $60 \%$ (16,6\%). Assim, a maior parte deles foi mantida após essa análise $(91,6 \%)$. Note-se que, nessa dimensão, todos os itens apresentaram concordância na dimensão para a qual, originalmente, foram desenvolvidos. Houve, também, três casos de ausência de classificação do juiz (itens 51, 37 e 59).

Por fim, a análise dos itens que compõem a dimensão Sentido da Vida indicou que cinco dos itens foram descartados por apresentarem concordância menor do que o valor desejado em mais de um dos três primeiros critérios analisados (itens 16, 7, 109, 106 e 65). A análise da dimensão a que pertencem, segundo julgamentos dos juízes, mostrou que somente os itens 30 e 78 apresentaram concordância adequada. Os demais apresentaram valores de $40 \%$ $(n=3)$ e $60 \%(n=3)$. Essa foi a dimensão que mais apresentou problemas em sua classificação. Além disso, vários juízes não conseguiram classificar um item em nenhuma das dimensões criadas e vários itens foram classificados na dimensão Aspirações Positivas. Optou-se por reescrever seis itens, pois eles apresentaram problemas somente na dimensão (itens 95, 91, 71, 33, 40 e 6).

Posteriormente, uma segunda rodada de avaliação dos juízes foi conduzida, visando o julgamento dos itens que precisaram ser reescritos. Os 14 itens foram enviados novamente para os mesmos juízes somente para que avaliassem o critério dimensão (Tabela 3). A coluna intitulada Dimensão Ideal representa a dimensão para o qual o item foi, originalmente, desenvolvido.

Tabela 3. Resultados da segunda rodada de análise dos juízes

\begin{tabular}{cccccccc}
\hline & \multicolumn{9}{c}{ Juiz } & & & Dimensão Ideal $\%$ de Concordância \\
\hline Item & 1 & 2 & 3 & 4 & 5 & 1 & 100 \\
91 & 1 & 1 & 1 & 1 & 1 & 7 & 80 \\
\hline
\end{tabular}




\begin{tabular}{|c|c|c|c|c|c|c|c|}
\hline 105 & 5 & 5 & 5 & 5 & 5 & 5 & 100 \\
\hline 55 & 4 & 4 & 4 & 4 & 7 & 4 & 80 \\
\hline 6 & 7 & 7 & 7 & 7 & 7 & 7 & 100 \\
\hline 108 & 1 & 5 & 1 & 1 & 5 & 1 & 60 \\
\hline 71 & 7 & 7 & 7 & 7 & 7 & 7 & 100 \\
\hline 43 & 4 & 5 & 4 & 4 & 4 & 4 & 80 \\
\hline 103 & 3 & 5 & 3 & 3 & 5 & 3 & 60 \\
\hline 25 & 1 & 1 & 1 & 1 & 4 & 1 & 80 \\
\hline 33 & 7 & 7 & 7 & 7 & 7 & 7 & 100 \\
\hline 44 & 7 & 7 & 4 & 7 & 7 & 7 & 80 \\
\hline 40 & 4 & 7 & 4 & 7 & 4 & 4 & 60 \\
\hline 99 & 5 & 2 & 5 & 5 & 5 & 2 & 80 \\
\hline
\end{tabular}

Nota. Na coluna Dimensão ideal: 1. Relacionamentos Afetivos; 2. Estudo; 3. Trabalho; 4. Aspirações Positivas; 5. Bens Materiais; 6. Religião/Espiritualidade; 7. Sentido da Vida.

Nota-se que, novamente, três itens apresentaram baixa concordância entre os juízes. Em relação ao item 99, que abordava a questão de ganhar dinheiro com a faculdade mesmo que isso não trouxesse realização pessoal, criado para a dimensão Estudo, o que se fez notar é que os juízes o avaliaram como pertencente à dimensão Bens Materiais. Diante desse fato, as autoras optaram por realocá-lo na dimensão sugerida pelos juízes. Dada a quantidade de itens e de dimensões avaliadas, um resumo dos resultados foi organizado na Tabela 4, a seguir.

Tabela 4. Número de itens por dimensão que apresentaram concordância inadequada, por critério considerado

\begin{tabular}{cccccc}
\hline \multirow{2}{*}{ Dimensão } & \multicolumn{5}{c}{ Concordância inadequada } \\
\cline { 2 - 6 } & clareza & $\begin{array}{c}\text { Pertinência } \\
\text { prática }\end{array}$ & $\begin{array}{c}\text { Pertinência } \\
\text { teórica }\end{array}$ & $\begin{array}{c}\text { Dimensão } \\
\text { ideal }\end{array}$ & $\begin{array}{c}\text { No de } \\
\text { itens } \\
\text { excluídos }\end{array}$ \\
\hline 1 & 1 & - & - & 3 & - \\
2 & 3 & 2 & 2 & 4 & 2 \\
3 & 1 & 2 & 2 & 4 & 2 \\
4 & 3 & 1 & 1 & 4 & 1 \\
5 & 3 & 3 & 3 & 1 & 2 \\
6 & 6 & 3 & 2 & 2 & 2 \\
7 & 3 & 5 & 8 & 10 & 5 \\
Total & 20 & 16 & 18 & 28 & 14 \\
\hline
\end{tabular}

Nota. Na coluna Dimensão: 1. Relacionamentos Afetivos; 2. Estudo; 3. Trabalho; 4. Aspirações Positivas; 5. Bens Materiais; 6. Religião/Espiritualidade; 7. Sentido da Vida.

A análise mostrou que, no primeiro filtro, diversos itens não satisfizeram o critério de concordância de $80 \%$ por parte dos juízes, sendo 20 deles em relação ao critério de clareza, 16 em relação à pertinência prática e 18 em relação à 
pertinência teórica, sendo importante destacar que um item pode ter apresentado resultados insatisfatórios em mais de um critério. Em relação à análise da dimensão, segundo filtro utilizado no estudo, 28 itens não foram corretamente classificados pelos juízes na dimensão para a qual foram, teoricamente, desenvolvidos. Aqueles casos em que problemas já foram detectados na primeira análise, foram excluídos, em um total de 14 itens. Outros 14 itens apresentaram problemas somente na dimensão, sendo reescritos e avaliados novamente pelos mesmos juízes. Somente três deles não obtiveram, novamente, classificação adequada, sendo assim excluídos da escala.

Dessa forma, a versão da EPVA enviada para os juízes continha 116 itens e, após a análise da porcentagem de concordância dos juízes, passou a ter 102 itens. Esses itens $(n=102)$ fizeram parte da análise do coeficiente de Kappa, com o objetivo de levantar dados acerca da qualidade do julgamento realizado por cada juiz. Para isso, cada juiz teve estimado três índices: o número de itens que classificou em cada dimensão, o número de acertos e porcentagem de acerto. Os dois últimos índices foram calculados a partir da comparação do julgamento realizado por cada juiz, com a classificação de um "juiz ideal" (dimensão para o qual o item obteve concordância na análise anterior - Tabela 3, coluna dimensão conferida). Os resultados dessa segunda análise encontram-se na Tabela 5.

Tabela 5. Estatística kappa para a avaliação dos juízes por dimensão

\begin{tabular}{|c|c|c|c|c|c|c|c|c|c|}
\hline \multirow{2}{*}{ Juiz } & \multirow{2}{*}{ Kappa } & \multirow{2}{*}{ Critério } & \multicolumn{7}{|c|}{ Dimensão } \\
\hline & & & 1 & 2 & 3 & 4 & 5 & 6 & 7 \\
\hline \multirow{3}{*}{1} & 0,910 & $\begin{array}{l}\text { Número de itens } \\
\text { classificados }\end{array}$ & 19 & 15 & 12 & 14 & 14 & 10 & 7 \\
\hline & $(p=0,033)$ & Acertos & 18 & 13 & 11 & 11 & 14 & 10 & 7 \\
\hline & & Porcentagem de acerto & 94 & 86 & 91 & 78 & 100 & 100 & 100 \\
\hline \multirow{3}{*}{2} & 0,909 & $\begin{array}{c}\text { Número de itens } \\
\text { classificados }\end{array}$ & 18 & 15 & 12 & 14 & 14 & 10 & 7 \\
\hline & $(p=0,033)$ & Acertos & 14 & 14 & 11 & 13 & 14 & 10 & 7 \\
\hline & & Porcentagem de acerto & 77 & 93 & 91 & 92 & 100 & 100 & 100 \\
\hline \multirow{3}{*}{3} & 0,809 & $\begin{array}{l}\text { Número de itens } \\
\text { classificados }\end{array}$ & 19 & 14 & 11 & 13 & 12 & 10 & 7 \\
\hline & $(p=0,046)$ & Acertos & 17 & 12 & 9 & 7 & 11 & 10 & 6 \\
\hline & & Porcentagem de acerto & 89 & 85 & 81 & 53 & 91 & 100 & 85 \\
\hline 4 & $\begin{array}{c}0,921 \\
(p=0,031)\end{array}$ & $\begin{array}{c}\text { Número de itens } \\
\text { classificados }\end{array}$ & 19 & 15 & 11 & 14 & 14 & 10 & 7 \\
\hline
\end{tabular}




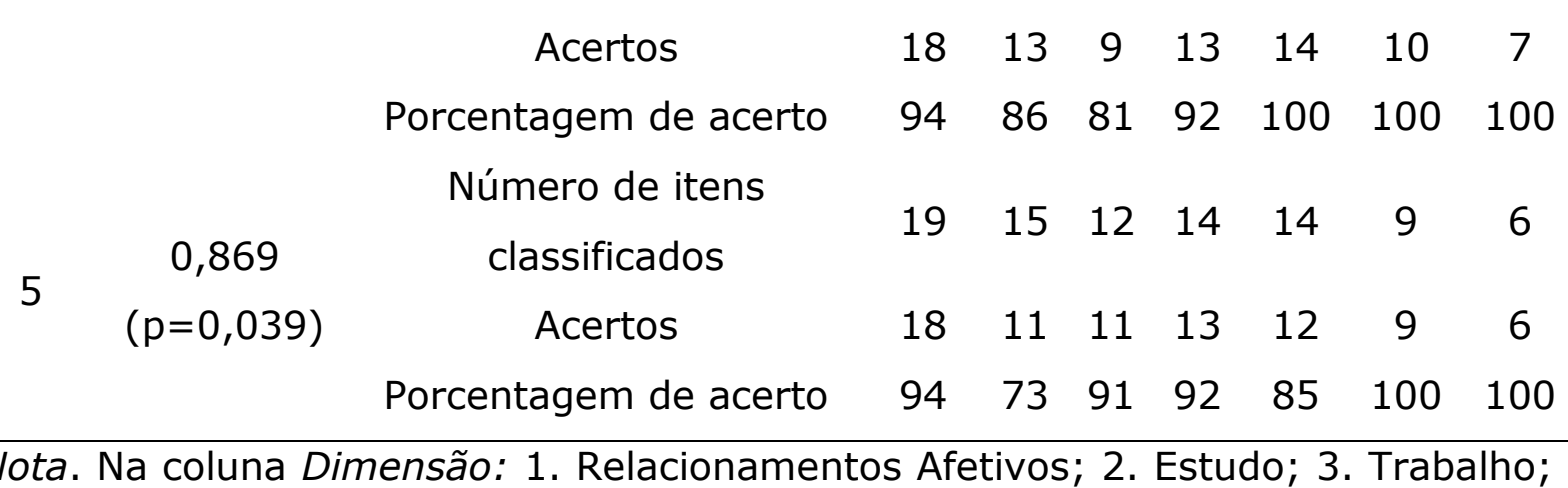

4. Aspirações Positivas; 5. Bens Materiais; 6. Religião/Espiritualidade; 7. Sentido da Vida.

Os resultados indicaram que todos os juízes obtiveram índice de concordância considerado excelente, ou seja, acima de 0,75 e $p \leq 0,05$. A dimensão 6, Religião/Espiritualidade, foi a que obteve maior porcentagem de acerto entre os juízes (100\% para todos os juízes), seguida da dimensão 7 , Sentido da Vida, que também teve alta porcentagem de acerto, sendo que 4 juízes obtiveram 100\% de concordância. A dimensão 4, Aspirações Positivas, foi a dimensão que demonstrou a menor porcentagem de acerto, sendo que um juiz obteve apenas $53 \%$.

\section{DISCUSSÃO}

A análise de conteúdo é uma maneira de avaliar os itens de um instrumento a partir do julgamento de especialistas em determinado construto (Alves, Souza \& Baptista, 2011; Beckstead, 2009). Tal método pode auxiliar o pesquisador, no momento de construção de um novo instrumento, sendo recomendada sua realização antes da coleta de dados empírica (Pasquali 2010). Tanto a avaliação dos juízes como o julgamento quanto à adequação da literatura devem ser realizados antes do instrumento ser aplicado à população alvo, o que torna a análise de conteúdo um procedimento primordial na construção de novas medidas, não devendo, em hipótese nenhuma, ser confundido como um procedimento menos rigoroso do que os demais tipos de evidências de validade (Alexandre \& Coluci, 2011; Slocurnb \& Cole, 1991).

De um modo geral, os resultados apontaram a adequação da escala às áreas que ela pretende avaliar. A maior parte dos itens foi classificada como adequada pelos juízes, tomando-se os critérios de clareza, pertinência teórica e pertinência prática (dos 116 itens julgados nesse três critérios, portanto 348 avaliações realizadas, 54 apresentaram concordância inadequada, ou seja, $15,5 \%)$. Em relação à dimensão, $27,4 \%(n=28)$ apresentaram tal resultado.

Analisando-se mais especificamente a dimensão, pode-se ver que a Sentido da Vida foi a que apresentou maiores problemas (16 itens não apresentaram concordância adequada em alguns dos três primeiros critérios), 
seguida da Religião/Espiritualidade $(n=11)$. Salienta-se que a primeira dimensão citada também foi a que mais apresentou discordância em relação à dimensão. Por outro lado, a dimensão Relacionamento Afetivos teve seus itens melhores avaliados pelos juízes. Nesse sentido, uma hipótese de que se levanta envolve a possibilidade de que a definição da dimensão Sentido de Vida, fornecida aos juízes, possa não ter sido clara o suficiente para embasar o julgamento ou ainda ser ampla demais e envolver outras dimensões, de modo a causar julgamentos inadequados. Pode-se pensar que a dificuldade dos juízes em avaliar tal dimensão está relacionada ao fato de que a mesma diz respeito a questões existenciais, ou seja, facetas muito mais difíceis de serem nomeadas, cuja interpretação é um processo mais subjetivo. Da mesma forma, operacionalizar essa dimensão em forma de itens foi também uma tarefa difícil. Foi, aparentemente fácil para os juízes, classificar o que é objetivo, como bens materiais ou outras pessoas (na dimensão relacionamentos afetivos).

Diante dos resultados, se torna preciso avaliar se os baixos índices de concordância dos juízes em alguns itens refere-se a dificuldades específicas em relação ao conteúdo dos itens que apresentaram problema ou ao domínio dos juízes na temática. $O$ requisito utilizado para seleção dos juízes (notadamente envolvendo o nível educacional), pode não ter garantido, por si só, que esses juízes serão adequados para avaliar um instrumento de uma temática que eles podem ter pouco conhecimento. Tal fato pode ter exercido importante influência nos resultados, sendo apontado como uma das limitações do estudo, sendo importante, no entanto, destacar o número reduzido de profissionais que atuam na temática dos projetos de vida em adolescentes no Brasil.

O número de itens é uma preocupação do pesquisador e depende da complexidade do construto (Pasquali, 2010). Assim, considerando-se que foram descritas sete dimensões para o construto projeto de vida, fez-se necessário criar, inicialmente, uma alta gama de itens. Dos 116 itens que compunham a versão inicial da EPVA, 14 itens foram excluídos, resultando em uma versão da escala composta por 102 itens espalhados em suas sete dimensões (Relacionamentos Afetivos $=21$; Estudo $=16 ;$ Trabalho $=12 ;$ Aspirações Positivas $=16 ;$ Bens Materiais $=15 ;$ Religião/Espiritualidade $=10$ e Sentido da $V i d a=12$ itens). Após duas rodadas de avaliação, foi possível manter $88 \%$ da escala inicial, o que nos permitiu concluir que os itens apresentaram boas evidências de validade de construto. Note-se que, nessa fase do estudo, as dimensões não apresentam um número homogêneo de itens, fato que não foi considerado, nessa fase do estudo, como objetivo das pesquisadoras para a construção da escala. 


\section{CONSIDERAÇÕES FINAIS}

De um modo geral, os resultados apresentados se mostraram positivos, indicando boas evidências de validade da escala, o que confirma a adequação do instrumento ao construto e dimensões que ele pretende avaliar. De um total de 116 itens iniciais, apenas 17 itens foram excluídos, sugerindo a necessidade de que os mesmos sejam reformulados posteriormente, caso haja a intenção de se aumentar o número de itens total da escala ou de alguma dimensão específica.

Torna-se relevante destacar que o estudo aqui apresentado constitui-se em um primeiro passo na busca por indicadores acerca das evidências de validade da escala em questão, sendo necessária a condução de outras investigações até que o instrumento possa atender aos requisitos do Conselho Federal de Psicologia e, consequentemente, ser disponibilizado para uso profissional. Como sugestão de futuros estudos, envolvendo a investigação das qualidades psicométricas, a busca por outras fontes de evidências de validade, bem como investigações acerca da sua precisão é recomendada. Tais estudos poderão incrementar as evidências positivas aqui apresentadas. Do mesmo modo, as evidências de validade de conteúdo poderão ser ampliadas a partir do emprego de outros métodos de estimativa, também indicados na literatura científica, tais como a razão da validade de conteúdo (RVC) ou ainda a validade aparente, destacadas por Pacico e Hutz (2015).

Ainda que os resultados tenham se mostrado positivos, recomenda-se cautela na interpretação dos dados aqui apresentados. Primeiramente por esse ser apenas o primeiro estudo conduzido com o instrumental. Segundo pelas limitações já indicadas anteriormente, notadamente relacionadas à seleção dos juízes e à descrição das dimensões fornecidas aos mesmos para a classificação dos itens. É possível que elas não tenham sido suficientes para que 0 juiz classificasse corretamente $o$ item.

Espera-se que esse estudo possa incentivar a condução de mais pesquisas relacionadas aos projetos de vida dos adolescentes e que, posteriormente, o instrumento possa ser utilizado não só com o objetivo de avaliação desses projetos mas, também, como ferramenta para a identificação de dimensões fortes e fracas no individuo. Assim, os resultados dela provenientes poderão ser utilizados para o planejamento de intervenções desenvolvidas para estimular o planejamento do futuro no adolescente. A EPVA será de grande utilidade para pesquisadores e profissionais da psicologia que visam estudar a adolescência contemporânea e seus projetos de vida, em especial, por ser um instrumento especificamente desenvolvido para a população brasileira e adequado à nossa realidade cultural. 


\section{DECLARAÇÃO DE CONFLITOS DE INTERESSE}

Não há conflitos de interesse.

\section{REFERÊNCIAS}

Alexandre, N. M. C., \& Coluci, M. Z. O. (2011). Validade de conteúdo nos processos de construção e adaptação de instrumentos de medidas. Ciência e Saúde, 16(7), 3061-3068. http://dx.doi.org/10.1590/S141381232011000800006.

Alves, G. A. S., Souza, M. S., \& Baptista, M. N. (2011). Validade e precisão de testes psicológicos. In R. A. M. Ambiel, I. S. A. Rabelo, S. V. Pancanaro, G. A. S. Alves, \& I. F. A. S. Leme (Eds.), Avaliação Psicológica: Guia de consulta para estudantes e profissionais de psicologia (pp. 109-128). São Paulo: Casa do Psicólogo.

Araújo, U. F., Arantes, V. A., Danza, H. C., Pinheiro,V.P. G., \& Garbin, M. (2016). Principles and methods to guide education for purpose: A Brazilian experience. Journal of Education for Teaching, 42(5), 556-564. https://doi.org/10.1080/02607476.2016.1226554.

Beckstead, J. W. (2009). Content validity is naught. International Journal of Nursing Studies, 46, 1274-1283. https://psycnet.apa.org/doi/10.1016/j.ijnurstu.2009.04.014.

Bock, A. M. M., \& Liebesny, B. (2003). Quem eu quero ser quando crescer: Um estudo sobre o projeto de vida de jovens em São Paulo. Em S. A, Ozella (Ed.), Adolescências construídas a visão da psicologia sócio-histórica (pp. 203-222). São Paulo: Cortez.

Bronk, C, K. (2014). Purpose in life. A critical component of optimal youth development. Netherlands: Springer.

Bundick, M., Andrews, M., Jones, A., Mariano, J. M., Bronk, K. C., \& Damon, W. (2006). Revised youth purpose survey. Stanford, CA: Stanford Center on Adolescence.

Cardoso, C. P., \& Cocco, M. I. M. (2003). Projeto de vida de um grupo de adolescentes à luz de Paulo Freire. Revista Latino-Americana de Enfermagem, 11(6), 778-785. http://dx.doi.org/10.1590/S010411692003000600012 .

Crumbaugh, J. C. (1968). Cross-validation of Purpose in Life test based on Frankl's concepts. Journal of Individual Psychology, 24, 74-81.

Crumbaugh, J. C., \& Maholick, L. T. (1964). An experimental study in existentialism: The psychometic approach to Frankl's concept of noogenic neurosis. Journal of Clinical Psychology, 20, 200-207. https://psycnet.apa.org/doi/10.1002/10974679(196404)20:2\%3C200::AID-JCLP2270200203\%3E3.0.CO;2-U. 
D'Aurea-Tardeli, D. (2008). A manifestação da solidariedade em adolescentes: um estudo sobre a personalidade moral. Psicologia: Ciência e Profissão, 28(2), 288-303.

D'Aurea-Tardeli. D. (2010). Identidade e adolescência: Expectativas e valores do projeto de vida. Revista Eletrônica Pesquiseduca, 2(3), 65-74. Recuperado em 29 de maio de 2019, de http://periodicos.unisantos.br/index.php/pesquiseduca/article/view/76/pdf $\underline{1 .}$

Damon, W. (1995). Greater expectations: Overcoming the culture ofindulgence in our homes and schools. New York: Free Press.

Damon, W. (2009). O que o jovem quer da vida? Como pais e professores podem orientar e motivar os adolescentes (J. Valpassos, Trad.). São Paulo, SP: Summus.

Damon, W., Menon, J. \& Bronk, K.C. (2003). The development of purpose during adolescence. Applied Developmental Science, 7, 119-128. https://doi.org/10.1207/S1532480XADS0703 2.

Dellazzana-Zanon, L. L. (2016). Avaliação das propriedades psicométricas da Escala de Projeto de Vida para Adolescentes (EPVA) e suas relações com felicidade subjetiva e comportamento de risco (Projeto de pesquisa aprovado). Programa de Pós-Graduação em Psicologia. Pontifícia Universidade Católica de Campinas.

Dellazzana-Zanon, L. L., \& Freitas, L. B. L. (2015). Uma revisão de literatura sobre a definição de projeto de vida na adolescência. Interação em Psicologia, 19(2), 281-292. http://dx.doi.org/10.5380/psi.v19i2.35218.

Erikson, E. (1976). Identidade, juventude e crise (2a ed.). Rio de Janeiro, RJ: Zahar. (Trabalho original publicado em 1968).

Farias Júnior, J. C., Nahas, M. V., Barros, M. V. G., Loch, M. R., Oliveira, E. S. A., ..., \& Lopes, A. S. (2009). Comportamentos de risco à saúde em adolescentes no Sul do Brasil: Prevalência e fatores associados. Revista Panamericana de Salud Pública, 25(4), 344-352. Recuperado em 29 de maio de 2019, de https://scielosp.org/pdf/rpsp/2009.v25n4/344-352/pt.

Feijó, R. B., \& Oliveira, E. A. (2001). Comportamento de risco na Adolescência. Jornal de Pediatria, 77(2), 125-134. http://dx.doi.org/10.2223/JPED.300.

Fleiss, J. L., Levin, B., \& Paik, M. C. (2003). The Measurement of Interrater Agreement. In J. L. Fleiss, B. Levin, \& M. C. Paik (Eds.), Statistical Methods for Rates and Proportions (pp. 598-626). New York, NY: John Wiley \& Sons Inc.

Furlani, D. D., \& Bomfim, Z. A. C. (2010). Juventude e afetividade: Tecendo projetos de vida pela construção dos mapas afetivos. Psicologia \& Sociedade, 22(1), 50-59. http://dx.doi.org/10.1590/S010271822010000100007. 
Gonçalves, H. S., Borsoi, T. S., Santiago, M. A., Lino, M. V., Lima, I. N., \& Federico, R. G. (2008). Problemas da juventude e seus enfrentamentos: Um estudo de representações sociais. Psicologia e Sociedade, 20(2), 217-225. http://dx.doi.org/10.1590/S0102-71822008000200009.

Graf, L. P., \& Diogo, M. F. (2009). Projeções juvenis: Visões ocupacionais e marcas de gênero. Revista Brasileira de Orientação Profissional, 10(1), 7182. Recuperado em 29 de maio de 2019, de http://pepsic.bvsalud.org/scielo.php?script=sci arttext\&pid=S167933902009000100009\&lng=pt\&tIng=pt.

Hablas, R., \& Hutzell, R. (1982). The Life Purpose Questionnaire: An alternative to the Purpose in Life test for geriatric, neuropsychiatric patients. In S. A. Wawrytko (Ed.), Analecta Frankliana: The proceedings of the First World Congress of Logotherapy: 1980 (pp. 211-215). Berkeley: Strawberry Hill.

Inhelder, B., \& Piaget, J. (1976). Da lógica da criança a lógica da adolescente: Ensaio sobre a construção das estruturas operatórias formais (D. M. Leite, Trad.). São Paulo: Pioneira. (Trabalho original publicado em 1958).

La Taille, Y de. (2009). Formação ética: Do tédio ao respeito de si. Porto Alegre, RS: Artmed.

Law, B. M. (2012). Psychometric properties of the existence subscale of the Purpose in Life questionnaire for Chinese adolescents in Hong Kong. The Scientific World Journal.

Leão, G., Dayrell, J. T., \& Reis, J. B. (2011). Juventude, projetos de vida e Ensino Médio. Educação \& Sociedade, 32(117), 1067-1084. ttp://dx.doi.org/10.1590/S0101-73302011000400010.

Miranda, F. H. F. (2007). Projetos de vida na adolescência: Um estudo na área da ética e da moralidade. (Dissertação de mestrado). Universidade Federal do Espírito Santo. Vitória, Brasil.

Pacico, C.J., \& Hutz, C.S. (2015). Validade. In C.S Hutz, D. R Bandeira, \& C.M Trentini. (Eds.), Psicometria (pp. 71-84). Porto Alegre, RS: Artmed.

Paredes, E. C., \& Pecora, A. R. (2004). Questionando o futuro: As representações sociais de jovens estudantes. Psicologia: Teoria e Prática, Edição Especial, 49-65. Recuperado em 29 de maio de 2019, de http://editorarevistas.mackenzie.br/index.php/ptp/article/view/1174/873.

Pasquali, L. (2010). Instrumentação psicológica: Fundamentos e práticas. Porto Alegre, RS: Artmed.

Perroca, M.G., \& Gaidzinski, R. R. (2003). Avaliando a confiabilidade interavaliadores de um instrumento para classificação de pacientes: Coeficiente Kappa. Revista Escola de Enfermagem USP, 37(1), 72-80. http://dx.doi.org/10.1590/S0080-62342003000100009.

Piaget, J. (2007). Seis estudos de Psicologia (24th ed.) (M. A. M. D'Amorim, \& P. S. L Silva, Trad.). Rio de Janeiro, RJ: Forense Universitária. (Original publicado em 1964). 
Pratta, E. M. M., \& Santos, M. A. (2007). Opiniões dos adolescentes do ensino médio sobre o relacionamento familiar e seus planos para o futuro. Paidéia, 17(36), 103-114. http://dx.doi.org/10.1590/S0103-863X2007000100010.

Santos, M. I. (2002). Projeto de vida e perspectivas futuras: Um estudo sobre as representações sociais do tempo futuro presente. (Dissertação de mestrado). Pontifícia Universidade Católica de São Paulo, São Paulo.

Slocurnb, E. M., \& Cole, F. L. (1991). A Practical Approach to Content Validity. Applied Nursing Research, 4(4), 192-200.

Sobre as autoras

Jessica Particelli Gobbo é mestre pela Pontifícia Universidade Católica de Campinas, com pesquisa financiada pela Capes. jessicaparticelli@hotmail.com Tatiana de Cassia Nakano é docente do curso de Pós-Graduação strictu sensu em Psicologia pela Pontifícia Universidade Católica de Campinas. Trabalha na linha de pesquisa Instrumentos e Processos em Avaliação Psicológica.

tatiananakano@hotmail.com

Letícia Lovato Dellazzana-Zanon é docente do curso de Pós-Graduação strictu sensu em Psicologia pela Pontifícia Universidade Católica de Campinas. Trabalha na linha de pesquisa Intervenções Psicológicas e Processos de Desenvolvimento Humano. leticiadellazzana@gmail.com

Declaração da contribuição das autoras

Este artigo é baseado em parte da Dissertação de Mestrado da primeira autora, realizada sob orientação da terceira. Esta Dissertação de Mestrado foi desenvolvida no Programa de Pós-Graduação em Psicologia da Pontifícia Universidade Católica de Campinas. Certificamos que todas as autoras participaram suficientemente do trabalho para tornar pública sua responsabilidade pelo conteúdo. Todas as autoras participaram da conceitualização, investigação e visualização do artigo; a segunda autora foi responsável pelas análises estatísticas aplicadas; a primeira autora fez a redação inicial do artigo (rascunho) e todas as autoras são responsáveis pela redação final (revisão e edição).

O presente trabalho foi realizado com apoio da Coordenação de Aperfeiçoamento de Pessoal de Nível Superior - Brasil (CAPES) - Código de Financiamento 001.

Recebido em: 13/02/2017

$1^{\text {a }}$ revisão em: 10/08/2017

Aceito em: 19/09/2017 